Editorial

\title{
Acknowledgement to Reviewers of Coatings in 2013
}

Coatings Editorial Office, MDPI AG, Klybeckstrasse 64, CH-4057 Basel, Switzerland

Published: 24 February 2014

The editors of Coatings would like to express their sincere gratitude to the following reviewers for assessing manuscripts in 2013:

Ahadi, Amir

Algahtani, Ali

All, Angelo Homayoun

Almtoft, Klaus

Ando, Yasutaka

Ball, Richard

Ban, Masahito

Carneiro, Joaquim

Chidambaram, Dev

Douroumis, Dennis

Duan, Fei

Emanuelsson, Emma

Fauchais, Pierre

Ferrari, Maurizio

Fortes, Luis

Foster, David G.

Frach, Peter

Gagliardi, Mariacristina

Georgiou, E. P.

Goforth, Andrea M.

Gustavsson, Fredrik

Hanaor, Dorian

Hoskovcová, Irena

Ivanova, Elena P.

Jackson, John K.
Jackson, R. W.

King, Peter C.

Koralage, Ranga

Li, K. C.

Li, Sunny R.

Lin, Jing-chie

Liu, Baoshun

Lowe, Todd

Maitz, M. F.

Mäkelä, Jyrki M.

Marken, Frank

Miritello, Maria

Mosquera, Maria

Niu, Yaran

Nohava, Jiri

Nolan, Michael

Parnell, Andrew J.

Rajadas, Jayakumar

Ramirez, Anibal

Reshetilov, Anatoly N.

Rizzello, Loris

Robinson, Hans D.

Rosa, Roberto

Röttger, A.

Routh, Alexander
Sagawa, Takashi

Saghir, Ziad

Saha, Gobinda

Scheller, Bruno

Schilardi, P. L.

Schweiger, Paul

Shen, Wei

Simonot, Lionel

Steele, Terry W. J.

Steinmetz, Nicole F.

Strzhemechny, Yuri

Tait, Jeffrey G.

Tsay, Chien-Yie

Tsukanov, Igor

Velasco, J. I.

Ward, L. P.

Wetzler, Modi

Yamada, Naoomi

Yoshioka, Yasuo

Zhang, Donghui

Zhang, Hui

Zhang, Tao

Zuo, Fan 
(C) 2014 by the authors; licensee MDPI, Basel, Switzerland. This article is an open access article distributed under the terms and conditions of the Creative Commons Attribution license (http://creativecommons.org/licenses/by/3.0/). 